\title{
Return to Work nach stationärer Rehabilitation - Varianten der Berechnung auf der Basis von Patientenangaben und Validierung durch Sozialversicherungs-Beitragszahlungen
}

\section{Return to Work after Inpatient Rehabilitation - Alternative Calculations on the Basis of Patient Data and Validation of Social Security Contributions}

Autoren

Institute
R. Nübling ${ }^{1}$, R. Kaluscha ${ }^{2}$, G. Krischak ${ }^{2}$, D. Kriz ${ }^{1}$, H. Martin ${ }^{3}$, G. Müller ${ }^{4}$, J. Renzland ${ }^{5}$, M. Reuss-Borst ${ }^{6}$, J. Schmidt ${ }^{1}$, U. Kaiser ${ }^{1,7}$, E. Toepler ${ }^{8}$

Die Institutsangaben sind am Ende des Beitrags gelistet
Schlüsselwörter

- Outcomeforschung

- Return to Work

- Katamnese

- Patient-Reported Outcomes (PRO)

- Rehabilitations-StatistikDatenbasis (RSD)

- Sozialversicherungsbeiträge

Key words

- outcomes research

- return to work

- follow-up

- patient-reported outcomes (PRO)

- rehabilitation-statisticaldatabase (RSD)

- social security contributions

eingereicht: $\quad$ 19.04.2016 angenommen: 08.09.2016

\section{Bibliografie}

DOI http://dx.doi.org/

10.1055/s-0042-117282

Phys Med Rehab Kuror 2016;

26: 293-302

(c) Georg Thieme Verlag KG

Stuttgart · New York

ISSN 0940-6689

Korrespondenzadresse

Dr. R. Nübling, Dipl.-Psych.

GfQG - Gesellschaft für

Qualität im Gesundheitswesen Wendtstraße 1

76185 Karlsruhe

nuebling@gfqg.de

www.gfag.de

\section{Zusammenfassung \\ $\nabla$}

Fragestellung: Return to Work (RTW) stellt ein wesentliches Outcomekriterium medizinischer Rehabilitationsmaßnahmen dar. In der Literatur werden vielfältige Möglichkeiten beschrieben, das Konstrukt RTW zu operationalisieren. In der vorliegenden Arbeit werden 4 Berechnungsvarianten untersucht.

Material und Methoden: Es werden Daten der „Reha-QM-Outcome-Studie“, eine naturalistische, multizentrische Beobachtungsstudie herangezogen; sie beinhaltet Selbstangaben der Patienten und Daten aus der Rehabilitations-Statistik-Datenbasis (RSD). Die Stichprobe für die vorliegende Fragestellung umfasst $n=2947$ Versicherte der DRV Baden-Württemberg, die 2011 in 21 Kliniken behandelt wurden und zum Zeitpunkt der Reha-Antragstellung erwerbstätig oder arbeitslos waren. Die Stichprobe war weitgehend repräsentativ für die Grundgesamtheit der ausgewählten Versicherten.

Ergebnisse: Der Anteil der Rehabilitanden, die ein Jahr nach der Reha wieder oder weiter erwerbstätig waren (RTW-Quote) liegt je nach Berechnungsvariante zwischen 46 und 83\%. ROC-Analysen zeigen, dass aus den RSD-Daten als Außenkriterium ermittelten Sozialversicherungsbeiträge hinsichtlich der einfacheren Berechnung besser differenzieren, was für die Validität dieser Varianten spricht.

Schlussfolgerungen: Für den Vergleich von RTW-Quoten ist die zugrunde gelegte Berechnungsvariante entscheidend; diese sollten auf der Basis sowohl weiterer, von Patienten berichteten Outcomes als auch konkreter Beitragsleistungen überprüft sein.

\section{Hintergrund $\nabla$}

Eine in Abgrenzung zur Akutversorgung zentrale Aufgabe der medizinischen Rehabilitation besteht in der Erhaltung bzw. der Wiedergewin-

\section{Abstract \\ $\nabla$}

Objectives: Return to Work (RTW) is an essential outcome criterion in medical rehabilitation. There are many ways to operationalize the construct RTW. In the present study 4 calculation variants are compared.

Methods: The data used are from the "Rehabilitation QM Outcome Study”, a naturalistic, multicenter observational study including self-reports of rehabilitation patients and data from the German Rehabilitation Statistics Database (RSD). The sample for the present issue includes $\mathrm{n}=2947$ insurants of the DRV Baden-Württemberg, treated at 21 hospitals in 2011. All were economically active at the time of the rehabilitation application, that is employed or unemployed. The sample was broadly representative for the selected total group of insurants.

Results: Depending on the type of calculation, the proportions of insurants who were still or again employed one year after rehabilitation (RTW rate) differed between 46 and $83 \%$. ROC analyzes show that social security contributions differentiate better in the simpler calculation variant, which speaks for the validity of these variants.

Conclusions: For the comparison of RTW rates the selected calculation variant is crucial; these should be validated on the basis of both other, patient-reported outcomes and concrete social contributions. 
erfolgreiche Wiedereingliederung in den Beruf. Die „Return-toWork (RTW)“-Rate stellt deshalb ein wesentliches Ergebniskriterium für die Bewertung der medizinischen Rehabilitation dar $[1,2]$.

Eine Übersicht über Messmöglichkeiten geben z. B. Wasiak et al. [3]. Sie kommen zu dem Schluss, dass in der RTW-Forschung nicht nur der Begriff RTW sehr unterschiedlich definiert, sondern auch eine Vielzahl von Instrumenten eingesetzt wird. Auch Schultz et al. [4] beklagen das weitgehende Fehlen einer klaren Definition von RTW. Young et al. [5] untersuchten in ihrem Review unterschiedliche Operationalisierungen von RTW, ebenso Steenstra et al. [6], die ebenfalls eine große Bandbreite von Verfahren fanden. Die RTW-Quoten variierten dementsprechend erheblich, sie lagen in dieser Metaanalyse zwischen 64 und $98 \%$. So können zur Messung von RTW unterschiedliche Datenquellen herangezogen werden, z.B. Selbstangaben der Patienten, Daten von Arbeitgebern, Routinedaten von Kranken- und/oder Rentenversicherungsträgern. Zudem können sich die Messungen auf den RTW-Prozess- und/oder das RTW-Ergebnis beziehen, sie können einen Status zu einem bestimmtem Messzeitpunkt (3, 6, $12,18,24, \ldots$ Monate) nach Krankheitseintritt, nach Eintritt der Arbeitsunfähigkeit oder auch nach einer Reha-Maßnahme erheben oder auch kumulativ einen entsprechenden Zeitraum nach den genannten Ereignissen einschließen. Inhaltlich werden als Kriterien u.a. genannt: Zeit der Abwesenheit von der Arbeit, Zeit der verringerten Produktivität, Zeit der Arbeit ohne funktionelle Einschränkungen, Dauer der Berufs- oder Arbeitsunfähigkeit, Dauer der Fehltage nach dem Zeitpunkt einer Verletzung oder Erkrankung, Zeit bis zum Wiedereintritt in den gleichen oder einen anderen Job, voller Wiedereintritt an den bisherigen Arbeitsplatz [6-12].

Wie Steenstra et al. [10] weisen auch Streibelt und Egner für die Medizinische Rehabilitation in Deutschland darauf hin, dass RTW-Ergebnisse u.a. von Messmethode, Messzeitpunkt und Stichprobe abhängen [2]. Nach Morfeld [13] wird RTW in der rehabilitationswissenschafltichen Literatur häufig mit dem bloßen Status der Erwerbstätigkeit oder Arbeitsfähigkeit gleichgesetzt. Er kritisiert, dass diese Kriterien den Aspekt einer dauerhaften beruflichen Wiedereingliederung zu wenig berücksichtigen. Bezugnehmend auf Bürger et al. [14] wird vorgeschlagen, zur Berechnung von RTW über den Erwerbsstatus hinaus folgende Kriterien für eine „stabile Wiedereingliederung“ einzubeziehen: a) Fehlzeiten unter 12 Wochen im Jahr nach der Reha, b) keinen Rentenantrag gestellt und c) kein Rentenantrag geplant [15]. Diese Berechnungsvariante wird auch als „Return to Work in good health" bezeichnet $[16,17]$. Die genannten Kriterien korrelieren positiv mit einer langfristigen beruflichen Wiedereingliederung. Unter Einbezug dieser 3 zusätzlichen Kriterien ergeben sich z.T. deutlich niedrigere RTW-Anteile $[15,16]$.

Streibelt und Egner schlagen die Berechnung von RTW auf der Basis von 2 zentralen Konzepten vor: das der Zeitpunktquote und das der kumulativen Zeitverlaufsquote [2]. Mit der Zeitpunktquote wird der aktuelle Status der Erwerbstätigkeit zum jeweiligen Messzeitpunkt (z.B. 1 Jahr nach Ende der Reha) erfasst. Die kumulative Zeitverlaufsquote berücksichtigt den gesamten Zeitraum nach einer Rehabilitationsmaßnahme, d.h. sie enthält auch Rehabilitanden, die im Zeitfenster seit Ende der Maßnahme erwerbstätig waren bzw. dies noch sind. Dabei wird jeweils der Anteil der Erwerbstätigen an der jeweiligen Gesamtstichprobe gemessen ( 1 = RTW, 0 = kein RTW). Die Zeitpunktquote ist dabei immer etwas niedriger als die Zeitverlaufsmethode, da in letzterer auch Rehabilitanden erfasst werden, die auch ver- gleichsweise kürzere Zeiträume nach der Rehabilitation erwerbstätig waren (vgl. auch [18]).

Aus den Berechnungsvarianten a) Status der Erwerbstätigkeit („einfache Berechnung“) vs. „in good health“ und Zeitpunkt vs. Zeitverlauf können für die jeweiligen Kombinationen 4 unterschiedliche RTW-Quoten berechnet werden. Zentraler Fokus der vorliegenden Arbeit ist der Vergleich dieser RTW-Quoten sowie die Abschätzung deren Validität. Besondere Berücksichtigung finden dabei Beitragsleistungen aus Beschäftigung, wie sie in der Rehabilitations-Statistik-Datenbasis zur Verfügung stehen $[19,20]$. Eine vergleichende Validierung unterschiedlicher RTWBerechnungsvarianten unter Einbezug von Beitragszahlungen sowie „patient reported outcomes“ (PRO; [21]) steht bisher noch aus.

Folgende Fragestellungen stehen im Fokus der vorliegenden Arbeit:

1. Wie hoch sind die RTW-Quoten auf Grundlage der 4 Messvarianten?

2. Wie hoch ist der Zusammenhang zwischen diesen 4 Varianten?

3. Wie hoch ist der Zusammenhang zwischen RTW und den Sozialversicherungsbeiträgen aus Erwerbstätigkeit? Für welche Berechnungsvariante ist der Zusammenhang am höchsten? Welche RTW-Variante wird durch die Beiträge am besten diskriminiert?

4. Durch welche Ausgangsvariablen können die RTW-Varianten vorhergesagt werden?

5. Wie korrelieren RTWs mit anderen, von Patienten berichteten Ergebnismaßen (PROs; u.a. Patientenzufriedenheit, Nutzenbeurteilung, gesundheitsbezogene Veränderungen)?

\section{Material und Methoden}

$\nabla$

Es handelt sich um eine multizentrische naturalistische Beobachtungsstudie mit einem Messzeitpunkt für die Patientenbefragung (1-Jahres-Katamnese) bzw. den Beobachtungszeiträumen 36, 24 und 12 Monate vor und 12 Monate nach der Reha für die RSD-Versichertendaten (s.u.). Zur Abbildung der Ergebnisqualität wurden im Rahmen der katamnestischen Befragung 2 Varianten der Veränderungsmessung eingesetzt: die direkte (dVM; direkte Veränderungseinschätzungen im Sinne von „verbessert/verschlechtert“) und die quasi-indirekte Veränderungsmessung (qVM; retrospektive Einschätzung des Prä-Zustandes bei Rehabeginn im Vergleich mit dem aktuellen Zustand bei Katamnese). Für die Routine-Daten der Rentenversicherung (RSD) liegen „echte“ Prä- und Post-Daten vor (indirekte Veränderungsmessung, iVM; vgl. ausführlicher [21-23]).

Das Untersuchungsdesign unterscheidet 3 Daten-Stränge, von denen für die vorliegende Arbeit die ersten beiden berücksichtigt werden:

- Katamnestische Befragung der Versicherten (Selbstangaben, PROs; Individualebene I).

- Versichertendaten der DRV Baden-Württemberg (RSD; Individualebene II)

- Kennzahlen aus dem einheitlichen Kennzahlensystem des Qualitätsmanagement des Klinikverbundes (Einrichtungsebene).

\section{Patientenbefragung}

Für die Befragung der Patienten wurde eine Adaptation des im Rahmen der EQUA-Studie [24] entwickelten Katamnesefragebogen (KFB) eingesetzt und in Teilbereichen durch weitere Verfahren (z. B. IMET, [25]) ergänzt. Der KFB erfasst u. a. subjektive Out- 
come-Parameter wie gesundheitliches Befinden, gesundheitliche Veränderungen, die Nutzenbeurteilung der Rehabilitation sowie arbeitsbezogene und gesundheitsökonomisch relevante Parameter. Integriert wurden u. a. folgende Skalen (vgl. ausführlicher [22,23]).

- Skala GBesc „Gesundheitliche Beschwerden“ (13 Items; Statuseinschätzungen; Cronbach's $\alpha=0,89$; Range Gesamtskala: 0-52 Skalenpunkte; hohe Werte= ausgeprägte Beschwerden) mit den Subskalen Körperliche Beschwerden $(\alpha=0,69)$ und Psychische Beschwerden $(\alpha=0,92)$; hohe Werte = ausgeprägte Beschwerden [22,25].

- Skala GB10 „Gesundheitliches Befinden“ (10 Items; Statuseinschätzungen; Cronbach's $\alpha=0,91$; Range $=10-60$; hohe Werte = gutes Befinden; [24]).

- VM8 Skala zur Erfassung subjektiv erlebter Veränderungen (8 Items; direkte Veränderungseinschätzungen; $\alpha=0,90-\alpha=$ 0,94; Range: 8-40; hohe Werte=viele positive Veränderungen; $[24,26])$.

- ZUF-8 ([28]) Skala zur Messung der allgemeinen Patientenzufriedenheit (8 Items; Range: 8-32, hohe Werte=hohe Zufriedenheit; $\alpha$ zwischen 0,82 (Schmerzpatienten) und 0,93 (Psychosomatik); [29]).

- MBOR-P, Kurzskala zur Einschätzung der beruflichen Schwerpunktsetzung der durchgeführten Rehabilitationsmaßnahme aus Patientensicht ( 7 Items; $\alpha=0,91$; Range $0-40$, hohe Werte $=$ starke Schwerpunktsetzung; [30]).

Die „einfache“ Berechnung des Anteils an Rehabilitanden, die zum Zeitpunkt der 1-Jahres-Katamnese (RTW-Zeitpunktquote) und des Anteils der Rehabilitanden, die im Zeitraum bis ein Jahr nach Entlassung erwerbstätig waren (kumulative Zeitverlaufsquote), erfolgte auf der Basis der folgenden Items; hierbei wurde jeweils der Anteil der Erwerbstätigen an der Gesamtstichprobe bestimmt (vgl. Streibelt \& Egner [2]).

- Die RTW-Zeitpunktquote wurde anhand des Items „Sind Sie derzeit erwerbstätig?“ ermittelt. Die Antwortalternativen ,ja, Vollzeit berufstätig“, „ja, Teilzeit berufstätig“, ,ja, in Ausbildung“ wurden dabei als 1 (RTW=1), die Alternativen „nein, arbeitslos“, „nein, Zeitrente“, „nein, dauerhaft berentet“ und „nein, ausschließlich Hausfrau/Hausmann“ als $0(\mathrm{RTW}=0)$ kodiert. Der Anteil der „ja“-Antwortalternativen (summierter Prozentanteil) entspricht der Zeitpunktquote.

- Die kumulative RTW-Zeitverlaufsquote wurde über das Item „Falls Sie vor der stationären Reha-Maßnahme berufstätig waren: Wann nach Abschluss der stationären Reha-Maßnahme haben Sie Ihre Berufstätigkeit wieder aufgenommen?“ mit den 3 Antwortalternativen „sofort“, „innerhalb von Tagen“ und „ich kann bis heute nicht mehr arbeiten“ berechnet. Hierbei wurden die ersten beiden Kategorien zusammengefasst $(\mathrm{RTW}=1)$ und der dritten $(\mathrm{RTW}=0)$ gegenübergestellt. Die Summe der Prozentanteile der ersten beiden Kategorien ergibt die kumulative Zeitverlaufsquote.
Diese beiden Quoten werden der von Morfeld [13,15] bzw. Streibelt et al. [17] vorgeschlagenen Berechungsmethode „Return to work in good health“ gegenübergestellt. Hierzu wurde als RTW zusätzlich zu den obigen Merkmalen definiert, wenn ein Rehabilitand 1) im Jahr nach der Reha weniger als 12 Wochen arbeitsunfähig war, 2) keinen Rentenantrag gestellt hatte oder 3) keine Intention geäußert hatte, einen Rentenantrag zu stellen (Item: „Überlegen Sie, einen Antrag auf Rente wegen verminderter Erwerbsfähigkeit (Frührente aus Gesundheitsgründen) zu stellen?“). Die Zeitpunkt- und die kumulative Verlaufsquote wurde dann ebenfalls für die „in good health“-Variante berechnet, d.h. im folgenden werden 4 Quoten betrachtet:

- die „einfache“ RTW-Zeitpunktquote (RTW_ZP)

- die „einfache“ kumulative RTW-Verlaufsquote (RTW_kumV)

- die RTW-Zeitpunktquote „in good health“ (RTW_ZPgh) und

- die kumulative RTW-Verlaufsquote „in good health“ (RTW_ kumVgh)

Darüber hinaus wurden Analysen 4 patientenberichtete Einschätzungen auf Einzelitemebene einbezogen: „Zielerreichung“ ( 1 =vollständig bis 5 = überhaupt nicht erreicht), „Nutzenbeurteilung“ ( 1 = kein bis 4 = großer Nutzen) „Zufriedenheit mit dem Behandlungsergebnis“ ( 1 =sehr zufrieden bis $5=$ sehr unzufrieden) sowie „berufliche Leistungsfähigkeit“ ( 1 =voll, 2 = eingeschränkt, 3 = gar nicht leistungsfähig; vgl. [22,23]).

\section{Sozialversicherungsbeiträge}

Die geleisteten Sozialversicherungsbeiträge entstammen der Rehabilitations-Statistik-Datenbasis (RSD) der Deutschen Rentenversicherung Baden-Württemberg. Es wurden unterschiedliche Beitragsarten in Monaten (im folgenden „SV-Beitragsmonate“) mit sozialversicherungspflichtiger Beschäftigung entsprechend $\odot$ Tab. 1 gewichtet (gewichtete Beiträge) und summarisch zu einem Index zusammengefasst.

Dabei wurde für jeden Versicherten aus dem individuellen Versicherungsverlauf die jeweiligen Beiträge je Monat gewichtet und daraus für die Jahres-Zeiträume die Anzahl an Beschäftigungs- bzw. Beitragsmonaten aus Erwerbstätigkeit berechnet (gewichteter, monatsbezogener Index x 12). Bei der Gewichtung wurde die gesamtgesellschaftliche Perspektive eingenommen, d.h. Sozialversicherungsbeiträge aufgrund des Bezugs von Sozialleistungen (Arbeitslosen-, Übergangs- oder Krankengeld) sowie Rente gingen mit einem Gewichtungsfaktor von 0 ein, da es sich hierbei lediglich um Umverteilungen zwischen den unterschiedlichen Zweigen der sozialen Sicherung handelt. Die Gewichtung erfolgte auf der Basis der mittleren monatlichen sozialversicherungspflichtigen Einkünfte in der RSD Baden-Württemberg (ca. € 1600). Damit entsprechen z.B. die Einkünfte für einen $400 €-J o b \quad 25 \%$ dieses durchschnittlichen Einkommens. Die RSD-Daten wurden von der DRV Baden-Württemberg in anonymisierter Form für alle Rehabilitanden des Erhebungszeitraumes für die o.g. Beobachtungszeiträume zur Verfügung gestellt.

Tab. 1 Index für die Beitragszahlungen der Versicherten aus Erwerbstätigkeit, Merkmale der Gewichtung des Index.

$\begin{array}{ll}\text { Gewichtung } & \text { Beschreibung } \\ \text { Gewicht } \mathbf{1} & \begin{array}{l}\text { Beiträge aufgrund von Beschäftigung, freiwillige Beiträge, selbständige Handwerker, usw.; Grundlage: durchschnittliche sozialversiche- } \\ \text { rungspflichtige Einküfte der Versicherten der DRV BW=ca. 1600€) }\end{array} \\ \text { Gewicht } \mathbf{0 , 5} & \text { Beschäftigung in der Gleitzone (d.h. Einkommen von 401 bis } 800 €) \\ \text { Gewicht } \mathbf{0 , 2 5} & \text { geringfügige Beschäftigung („400€-Jobs“) } \\ \text { Gewicht } \mathbf{0} & \text { keine Beiträge, Übergangs-/Krankengeld, Arbeitslosengeld I/II oder Rente }\end{array}$




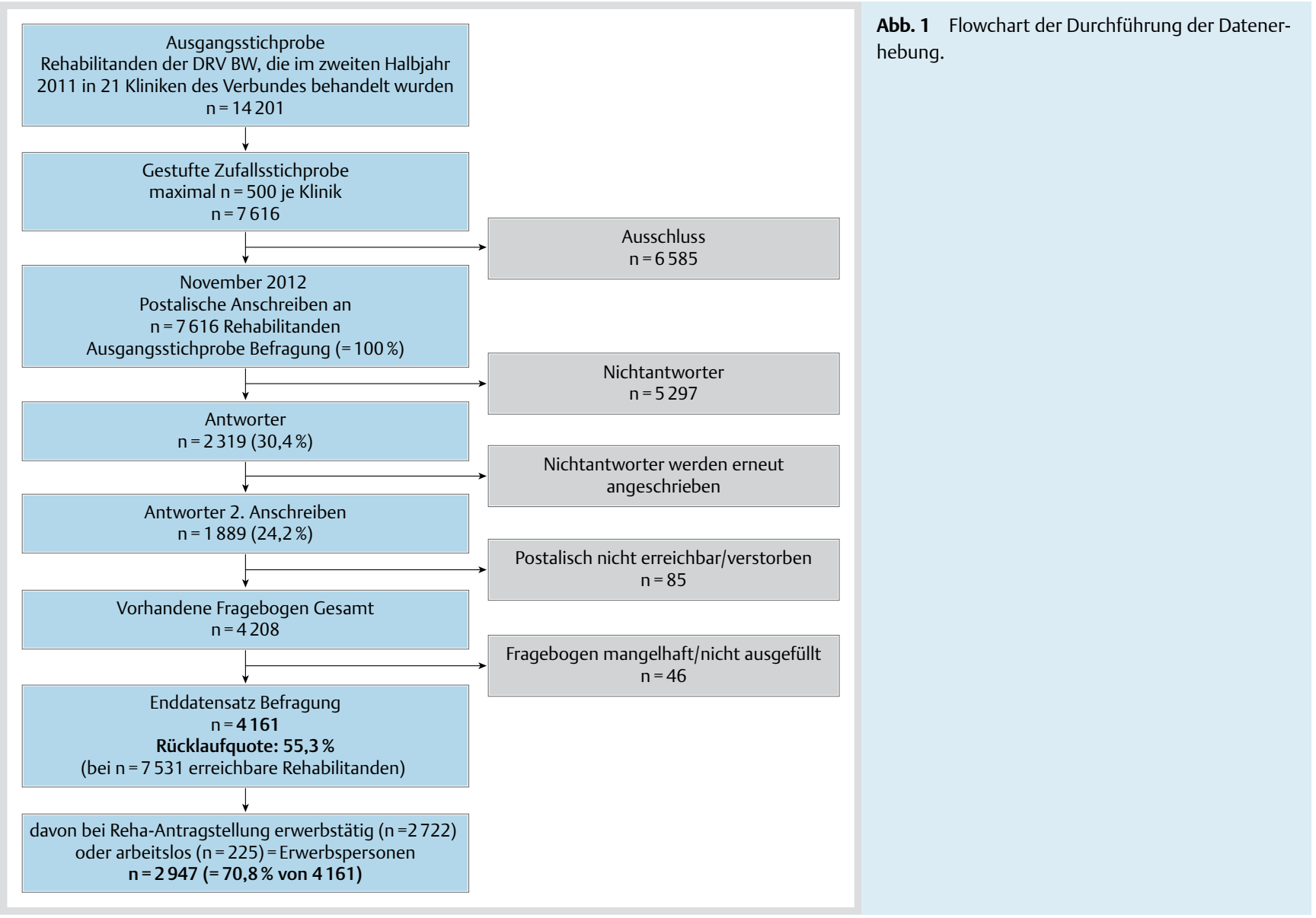

\section{Datenschutz}

Für die Verknüpfung der beiden Datensätze „katamnestische Patientenbefragung“ und „RSD“ wurde gemeinsam mit dem Datenschutzbeauftragten der DRV Baden-Württemberg ein spezielles Datenschutzkonzept entwickelt. Dazu wurde der Katamnesefragebogen mit einem eingedruckten Pseudonym an die ausgewählte Rehabilitandenstichprobe versandt. Beigelegt wurde eine ausführliche Aufklärung über die Studie und die geplante Verwendung der anonymisierten Daten. Bei Bereitschaft zur Teilnahme sandten die angeschriebenen Rehabilitanden den Fragebogen anonym an die Forschungsgruppe und erteilten damit eine konkludente Einwilligung. Gleichzeitig wurde das Pseudonym durch die DRV Baden-Württemberg in den RSD-Satz aufgenommen, der ansonsten keine Merkmale zur Personenidentifikation enthielt. So konnten Fragebogen- und RSD-Datensatz korrekt zusammengeführt werden, während zugleich die Identität der Teilnehmer gegenüber der Forschungsgruppe und ihre Fragebogenangaben gegenüber Rentenversicherung und Rehabilitationseinrichtungen geschützt blieben (siehe ausführlich [22]).

\section{Datenerhebung}

Einbezogen wurden erwachsene Rehabilitanden der DRV-Baden-Württemberg aller Indikationsbereiche (Ausnahme: Abhängigkeitserkrankungen), die im 2. Halbjahr 2011 in 21 Kliniken des Qualitätsverbunds Gesundheit (www.qualitaetsver bund-gesundheit.de) behandelt wurden. Aus der Gesamtmenge von $n=14201$ wurde eine gestufte Zufallsstichprobe von $\mathrm{n}=7616$ Versicherten gezogen. Je Klinik bzw. Fach-Abteilung wurden maximal $n=300$ Rehabilitanden per Zufall ausgewählt.
Bezogen auf Kliniken bzw. Abteilungen, in denen im genannten Zeitraum weniger als 300 Rehabilitanden der DRV Baden-Württemberg behandelt wurden, wurden alle Rehabilitanden einbezogen. Dadurch konnte gewährleistet werden, dass auch Verbundkliniken mit geringerem Anteil Versicherter der DRV Baden-Württemberg mit ausreichend hohen Patientenzahlen in die Studie eingehen konnten, um eine gute Grundlage für das klinikvergleichende Benchmarking [22] zu erhalten. Der postalische Fragebogenversand erfolgte durch die DRV Baden-Württemberg. Dort gingen auch die zurückgesandten Fragebögen ein, welche ungeöffnet an das auswertende Institut übergeben wurden, sodass die DRV Baden-Württemberg von den Antworten der Befragten keine Kenntnis erlangte. Von den angeschriebenen Patienten antworteten 4161; nach Abzug postalisch nicht erreichbarer sowie verstorbener Patienten lag die Rücklaufquote bei 55\%. Zum Zeitpunkt der Antragstellung der Rehabilitationsmaßnahme waren 2947 Patienten (ca. 71\% der Katamneseteilnehmer) erwerbstätig (2722) oder arbeitslos (225), d. h. Erwerbspersonen. Die nachfolgenden Analysen beziehen sich auf diese Teilstichprobe der Erwerbspersonen bei Antragstellung $\bigcirc$ Abb. 1.

\section{Repräsentativität der Katamnesestichprobe}

Zur Abschätzung der Repräsentativität wurde eine Non-Responder-Analyse auf der Basis der RSD-Daten durchgeführt. Im Ergebnis antworteten ältere Befragte und Befragte mit Erwerbstätigkeit im Jahr vor ihrer Rehabilitation etwas häufiger, ausländische Staatsangehörige und Rehabilitanden im § 51-Verfahren (Aufforderung zur Rehabilitation durch die Krankenkasse) deutlich und AHB-Patienten etwas seltener. Die sozialmedizinische Belastung, Geschlecht und eine Reihe weiterer Variablen (z.B. 
Diagnosegruppen, gewichtete Beschäftigungsmonate) hingegen beeinflussen die Teilnahmebereitschaft nicht. Die Studienstichprobe ist damit weitgehend repräsentativ für die Gesamtgruppe der angeschriebenen sowie der in den 21 Einrichtungen behandelten Patienten (siehe ausführlich [22]).

\section{Statistische Methoden}

Die Auswertung umfasste deskriptive sowie inferenzstatistische Berechnungen. Zur Abschätzung der Diskriminationsfähigkeit der RTW-Quoten wurden ROC-Analysen („Receiver Operating Characteristic“ [30]) durchgeführt. Zur Bestimmung von Zusammenhängen wurden Phi-Koeffizienten sowie binär logistische Regressionsanalysen berechnet.

Tab. 2 Stichprobenmerkmale der katamnestischen Antworter, die zum Zeitpunkt der Antragstellung Erwerbspersonen waren, Fragebogen- und RSD-Daten; $\mathrm{n}_{\max }=2947$.

\begin{tabular}{|c|c|c|}
\hline Merkmal/Variable & $\mathbf{n}$ & $\%$ \\
\hline Geschlecht ${ }^{1}$ & 2947 & \\
\hline weiblich & 1067 & 36,2 \\
\hline männlich & 1880 & 63,8 \\
\hline Familienstand ${ }^{1}$ & 2914 & \\
\hline ledig & 360 & 12,4 \\
\hline verheiratet & 1926 & 66,1 \\
\hline getrennt/geschieden & 527 & 18,1 \\
\hline verwitwet & 101 & 3,5 \\
\hline Schulabschluss ${ }^{1}$ & 2851 & \\
\hline bis Hauptschule & 1867 & 65,5 \\
\hline Realschule, Mittlere Reife & 711 & 24,9 \\
\hline Fachhochschulreife, Abitur & 232 & 7,9 \\
\hline Sonstiges & 41 & 1,4 \\
\hline Berufsabschluss ${ }^{1}$ & 2699 & \\
\hline kein Berufsabschluss & 389 & 14,4 \\
\hline Lehre & 1680 & 62,2 \\
\hline Fach-/Meisterschule & 453 & 16,8 \\
\hline Hochschule & 89 & 3,3 \\
\hline Sonstiges & 88 & 3,3 \\
\hline Letzte berufliche Stellung ${ }^{1}$ & 2717 & \\
\hline nicht erwerbstätig & 221 & 8,1 \\
\hline Auszubildender & 13 & 0,5 \\
\hline un-/angelernter Arbeiter & 663 & 24,4 \\
\hline Facharbeiter, nicht selbstständiger Handwerker & 884 & 32,5 \\
\hline Angestellter & 716 & 26,4 \\
\hline Selbstständiger & 93 & 3,4 \\
\hline Sonstiges & 127 & 4,7 \\
\hline Indikationsgruppen (Erstdiagnose) $)^{1,2}$ & 2947 & \\
\hline Kardiologie & 373 & 12,6 \\
\hline Onkologie & 371 & 12,7 \\
\hline Orthopädie & 1325 & 45,0 \\
\hline Psychosomatik & 337 & 11,4 \\
\hline Sonstige & 541 & 18,4 \\
\hline Belastung durch gesundheitliche Probleme ${ }^{1}$ & 2861 & \\
\hline extrem stark/stark & 2607 & 92,6 \\
\hline weniger stark/überhaupt nicht & 209 & 7,4 \\
\hline Erstauftreten der Krankheitsbeschwerden ${ }^{1}$ & 2875 & \\
\hline bis 2 Jahre vor Reha & 1474 & 51,3 \\
\hline 3-5 Jahre vor Reha & 521 & 18,1 \\
\hline mehr als 5 Jahre vor Reha & 880 & 30,6 \\
\hline Arbeitsunfähigkeitszeiten 12 Monate vor der Reha² & 2947 & \\
\hline Keine & 371 & 12,6 \\
\hline 1 bis unter 3 Monate & 1734 & 58,8 \\
\hline 3 bis unter 6 Monate & 392 & 13,3 \\
\hline \multirow[t]{2}{*}{6 Monate und mehr } & 450 & 15,3 \\
\hline & \multicolumn{2}{|r|}{$M(s d)$} \\
\hline Altersdurchschnitt bei Rehabeginn ${ }^{1}$ & 2947 & $51,7(8,1)$ \\
\hline Reha-Dauer in Tagen ${ }^{2}$ & 2947 & $23,8(5,0)$ \\
\hline
\end{tabular}

M: Mittelwert, sd: Standardabweichung; Datenquelle: ${ }^{1}$ Fragebogendaten, ${ }^{2}$ RSD

\section{Ergebnisse \\ $\nabla$}

\section{Katamnesestichprobe}

- Tab. 2 fasst ausgewählte deskriptive Stichprobenkennwerte für die untersuchte Stichprobe bei Beginn der Rehabilitation zusammen.

\section{Return to Work (RTW)}

- Abb. 2 zeigt die RTW-Quoten für die untersuchte Stichprobe nach den oben unterschiedenen 4 Berechnungsvarianten. Der Anteil wieder oder weiterhin erwerbstätiger Rehabilitanden liegt für die „einfache“ Berechnung zwischen 75 (Zeitpunkt) und 83\% (Zeitverlauf), nach der Variante „in good health“ zwischen 46 und $51 \%$. In die „in good health“-Variante ging dabei ein, dass $16,5 \%$ der Rehabilitanden im Jahr nach der Maßnahme länger als 12 Wochen arbeitsunfähig waren, 12,4\% einen Antrag auf Frühberentung gestellt hatten und $26,4 \%$ angaben, dass sie überlegt hätten, einen Antrag auf Frühberentung zu stellen.

\section{Zusammenhang zwischen den RTW-Quoten}

In $\odot$ Tab. 3 werden die Phi-Koeffizienten zwischen den 4 RTW's berichtet. Der höchste Zusammenhang besteht zwischen den beiden „in good health“-Varianten mit r=0,94. Der Zusammenhang zwischen den beiden „einfachen“ RTW-Varianten liegt mit $r=0,79$ etwas niedriger. Die Korrelationen zwischen RTW „in good health" und RTW liegen zwischen $r=0,45$ und $r=0,63$. Alle Zusammenhänge sind auf dem $1 \%$-Niveau signifikant.

\section{RTW-Quoten und Beitragszahlungen}

Im Folgenden wird der Index der gewichteten Beitragsmonate den jeweiligen RTW's gegenübergestellt. Die RSD-Daten lagen für alle hier betrachteten $n=2947$ Rehabilitanden vor. $\odot$ Abb. 3 und $\odot$ Abb. 4 zeigen den Verlauf der Beiträge in den 3 Jahren vor und einem Jahr nach der Reha-Maßnahme für Rehabilitanden mit positivem $($ RTW = 1 ) und negativem RTW $(R T W=0)$, d. h. gelungener und nicht gelungener Wiedereingliederung, jeweils für die „einfach“ berechnete RTW-Variante (links) und für die Variante RTW „in good health“ (rechts). In $\bullet \mathbf{A b b} . \mathbf{3}$ sind die beiden Zeitpunktquoten, in $\bullet$ Abb. 4 die beiden kumulativen Verlaufsquoten dargestellt.

Hierbei zeigt sich für die Rehabilitanden mit positivem RTW ein annähernd konstanter Verlauf der gewichteten Beitragsmonate zwischen ca. 11 und ca. 10 Monaten. Für Rehabilitanden, die

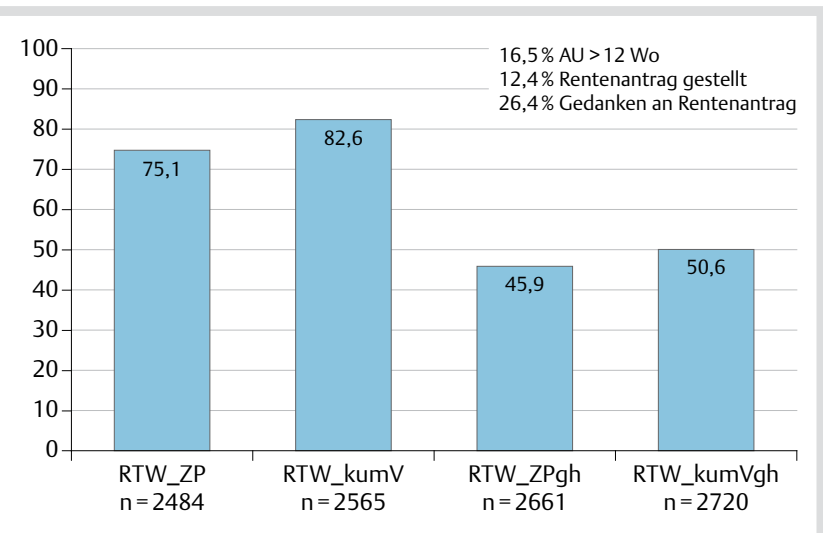

Abb. 2 Return to Work, Erwerbstätigkeit aktuell (1 Jahr nach der Reha-Maßnahme, Zeitpunktmethode; ZP) sowie im Jahres-Zeitraum nach Ende der Reha (Methode des kumulativen Zeitverlaufs; kumV) in Prozent; Patientenangaben; Vergleich „einfache“ vs. „in good health“ (gh)-Berechnung. 


\begin{tabular}{|c|c|c|c|c|}
\hline Merkmal/Variable & RTW_ZP & RTW_kumV & RTW_ZPgh & \multirow{5}{*}{$\begin{array}{l}\text { Tab. } 3 \text { Korrelationen zwischen } \\
\text { den RTW-Berechnungsvarianten; } \\
\text { Phi-Koeffizient, } \mathrm{n}_{\max }=2400 \text {. }\end{array}$} \\
\hline RTW_ZP: Zeitpunktquote & 1,000 & & & \\
\hline RTW_kumV: kumulative Verlaufsquote & 0,785 & 1,000 & & \\
\hline RTW_ZPgh: Zeitpunktquote „good health“ & 0,632 & 0,449 & 1,000 & \\
\hline RTW_kumVgh: kumulative Verlaufsquote „good health“ & 0,469 & 0,515 & 0,944 & \\
\hline
\end{tabular}
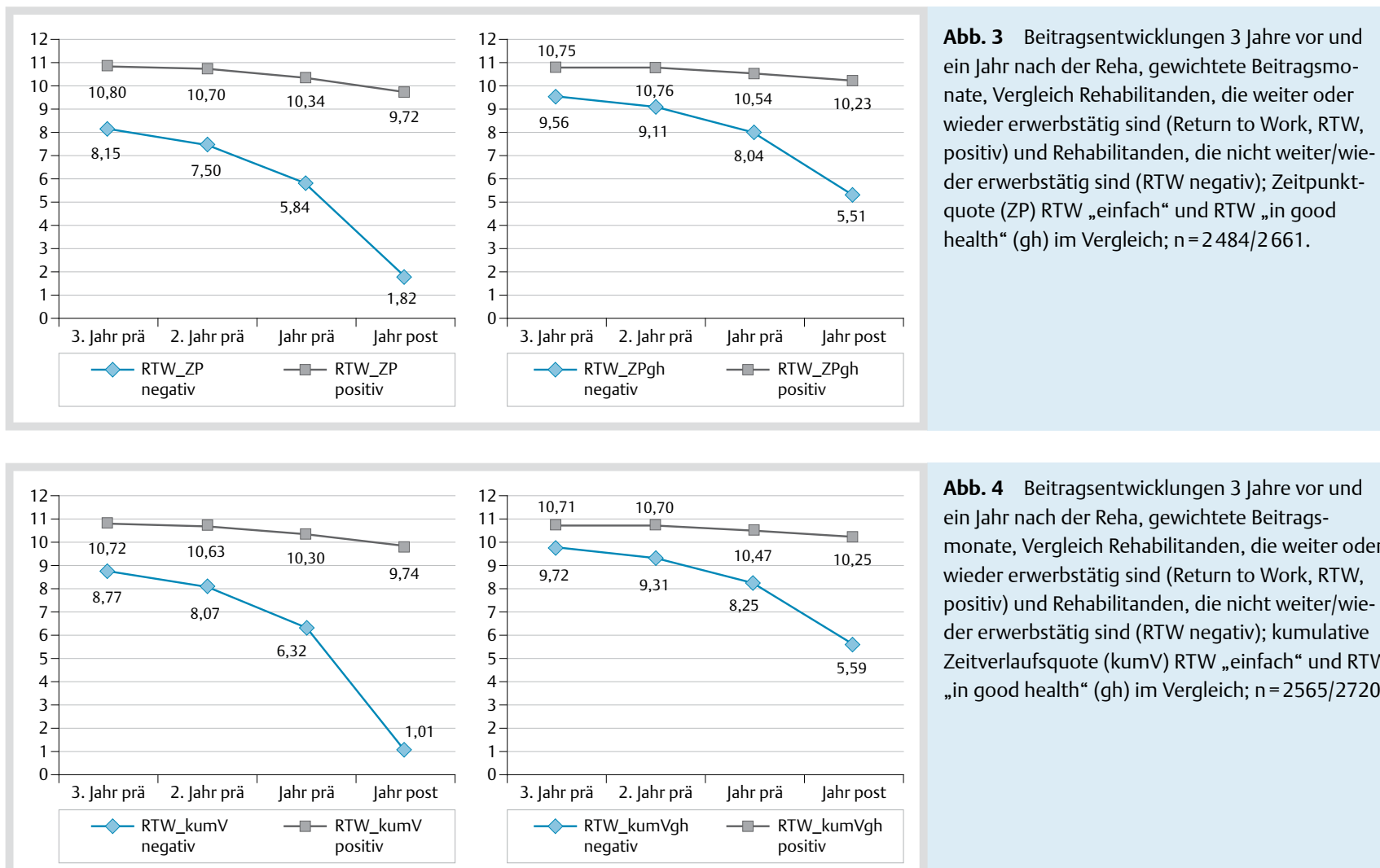

Abb. 4 Beitragsentwicklungen 3 Jahre vor und ein Jahr nach der Reha, gewichtete Beitragsmonate, Vergleich Rehabilitanden, die weiter oder wieder erwerbstätig sind (Return to Work, RTW, positiv) und Rehabilitanden, die nicht weiter/wieder erwerbstätig sind (RTW negativ); kumulative Zeitverlaufsquote (kumV) RTW „einfach“ und RTW "in good health“ ( $g$ h) im Vergleich; $n=2565 / 2720$.

nicht weiter/wieder erwerbstätig sind, also mit negativer RTW-Quote, ergibt sich für die „einfache“ Berechnungsvariante eine deutliche Reduktion der Beiträge, vor allem im Jahr nach der Reha-Maßnahme ( $\bullet$ Abb. 3). Demgegenüber ergibt sich für die ,in good health“-Variante ein sichtbar geringerer Differenzierungsgrad, der sich bereits im dritten Jahr vor der Reha andeutet. Ein ähnliches Bild ergibt sich auch für die beiden Quoten auf der Basis des kumulativen Zeitverlaufs ( 0 Abb. 4).

\section{Diskrimination der RTW-Varianten durch Beitragszahlungen}

In einem weiteren Schritt wurden zur Abschätzung der Diskriminationsfähigkeit (Sensitivität und Spezifität) der Beitragsmonate bezogen auf die 4 RTW-Berechnungsvarianten ROC-Analysen [31] gerechnet. Mit einer ROC-Analyse lässt die Validität eines Instruments oder eines Differenzierungsmerkmals überprüfen. Die ROC-Kurve liefert eine grafische Darstellung der Sensitivität und Spezifität eines Merkmals, ihr Verlauf beschreibt die Diskriminationsfähigkeit eines Merkmals in Bezug auf ein Außenkriterium. Im Rahmen der ROC-Analyse wird darüber hinaus die „Fläche unter der Kurve“ (Area under the curve, AUC) berechnet, der AUC-Wert liegt dabei zwischen 0,5 (auf Zufall beruhende Diskriminationsfähigkeit) und 1,0 (perfekte Diskriminationsfähigkeit).

In der vorliegenden Analyse wurden die RTW-Varianten dem Beitragsindex in Monaten gegenübergestellt. Die wichtigsten Ergebnisse sind in $\odot$ Tab. 4 zusammengefasst. Wie zu sehen, liegt die AUC zwischen 0,762 und 0,928, die Sensitivität zwischen 73,2 und $88,7 \%$ und die Spezifität zwischen 71,2 und $90,1 \%$. Am besten differenzierten die Beitragsmonate hinsichtlich der „einfach" berechneten kumulativen Verlaufsquote, während die beiden Varianten nach der „in good health“-Berechnung deutlich schlechtere Werte aufweisen. Die „einfache“ Zeitpunktquote liegt leicht unter der kumulativen Verlaufsquote.

\section{Vorhersage der RTW-Varianten durch Ausgangsvariablen}

- Tab. 5 zeigt die Odds-Ratios und deren p-Wert sowie die multiplen Zusammenhänge (Nagelkerke's $\mathrm{R}^{2}$ ) der auf der Basis von Selbstangaben (überwiegend retrospektiv) erhobenen und in das Modell einbezogenen Ausgangsmerkmalen in Bezug auf die 4 RTW-Berechnungsvarianten.

Statistisch bedeutsame Odds zeigten sich vor allem für Alter, Krankschreibungen unmittelbar vor sowie in den 12 Monaten vor der Reha, Arbeitslosigkeit vor der Reha sowie der Einschätzung der beruflichen Leistungsfähigkeit bei Rehabeginn. Darüber hinaus ergab sich auch für Rehabilitanden, die die RehaMaßnahme auf eigenen Wunsch veranlasst hatten, eine höhere Wahrscheinlichkeit, an den Arbeitsplatz zurückzukehren. Hinsichtlich des multiplen Zusammenhangs (Nagelkerke's $\mathrm{R}^{2}$ ) zeigt sich, dass die „einfach“ berechneten RTW's durch die einbezogenen Ausgangsvariablen etwas besser vorhergesagt werden können als die RTW's der „in good health“-Variante. Die Varianzauf- 
Tab. 4 ROC-Analysen SV-Beiträge in Monaten im Jahr nach der Rehamaßnahme für die RTW-Berechnungsvarianten.

\begin{tabular}{|c|c|c|c|c|c|c|}
\hline Merkmal/Variable & AUC Area under Curve & $\mathbf{n}$ & Signifikanz & Sensitivität & Spezifität & Cut-Off Monate \\
\hline RTW_ZP: Zeitpunktquote & 0,895 & 2484 & $<0,001$ & 85,6 & 85,6 & 5,52 \\
\hline RTW_kumV: kumulative Verlaufsquote & 0,928 & 2565 & $<0,001$ & 88,7 & 90,1 & 3,12 \\
\hline RTW_ZPgh: Zeitpunktquote „goodhealth“ & 0,764 & 2661 & $<0,001$ & 73,2 & 71,6 & 10,04 \\
\hline RTW_kumVgh: kumulative Verlaufsquote „goodhealth“ & 0,762 & 2720 & $<0,001$ & 73,6 & 71,2 & 11,04 \\
\hline
\end{tabular}

klärung liegt zwischen 28 und 43\%, der Anteil richtiger Klassifikationen zwischen 70 und $89 \%$.

\section{Zusammenhang mit Outcome-Variablen}

In $\odot$ Tab. 6 zeigt die Odds-Ratio-Schätzer für die 4 RTW-Varianten unter Einbezug subjektiver, von Patienten berichteten Outcomevariablen (PRO). Betrachtet wurden die Skalen ZUF8, MBOR-P, GB10, GBesc und VM8 sowie die Nutzenbewertung der Rehamaßnahme.

Hier waren die Zusammenhänge moderater. Die Odds-Ratios liegen zwischen $\mathrm{OR}=0,84$ und $\mathrm{OR}=1,31$, wobei die Skalen Gesundheitliches Befinden und die Skala VM8 (direkte Veränderungseinschätzung) für alle RTW-Berechnungsvarianten signifikant sind. Die multiple Korrelation mit den genannten Outcomemerkmalen als Prädiktoren und den 4 RTW's als Kriterien ergab eine Varianzaufklärung von 16 bis $22 \%$ (Nagelkerke's $\mathrm{R}^{2}$ ). Die Klassifikationsergebnisse waren dabei für die beiden einfacheren Berechnungsvarianten deutlich höher als bei den „in good health"-Varianten.

\section{Diskussion}

$\nabla$

Die Effektivität der Rehabilitation in Bezug auf die Sicherung der Erwerbsfähigkeit kann u.a. über die (Wieder-)Eingliederung in den Beruf (Return to Work, RTW) abgebildet werden. Für die Berechnung der RTW-Quote wurden unterschiedliche Varianten vorgeschlagen, eine der aktuellsten Systematiken schlägt vor, zwischen den Methoden Zeitpunkt und kumulativer Zeitverlauf zu unterscheiden [2]. Darüber hinaus wurde diskutiert, dass für die Abschätzung einer dauerhaften beruflichen Wiedereingliederung zusätzlich zu den Angaben der aktuellen oder auch zeitweisen Erwerbstätigkeit auch die Arbeitsunfähigkeitszeiten nach der Reha ( $<12$ Wochen), eine ggf. eingeleitete Antragstellung sowie die geäußerte Intension zur Frühberentung einbezogen werden müsste $[14,15,17]$. Diese Variante wurde u.a. als „Return to Work in good health“ bezeichnet; sie wurde in der Vergangenheit häufig als die validere Schätzung für RTW angesehen [15].

In der vorliegenden Arbeit wurden 4 Bestimmungsvarianten von RTW gegenübergestellt: die Zeitpunktquote und die kumulative Verlaufsquote, jeweils in „einfacher“ Berechnung sowie in der Variante ,in good health“. Die hieraus resultierenden RTW-Quoten unterscheiden sich dabei in der herangezogenen Stichprobe der Reha-QM-Outcome-Studie deutlich: hinsichtlich Zeitpunkt liegen sie bei $46 \%$ (,in good health“) sowie bei $75 \%$ („einfache“ Berechnung), hinsichtlich kumulativen Zeitverlauf zwischen 51 und $83 \%$.

Wie gezeigt wurde, korrespondieren die einfachen, ohne zusätzlichen Merkmale berechneten RTW's sehr gut mit den Beitragsverläufen, d.h. bei „Rückkehrern“ an den Arbeitsplatz (RTW=1) bleiben die Beiträge weitgehend konstant, bei „Nicht-Rückkehrern“ $(R T W=0)$ reduzieren sie sich hingegen stark. Die Unter- schiede der Beitragsverläufe sind bei der „in good health“-Methode nicht so eindeutig. Dieses Ergebnis wird unterstrichen durch ROC-Analysen, die zeigen, dass die Beitragsmonate bei den einfachen RTW's deutlich besser diskriminieren als für die RTW's nach der „in good health“-Variante. Dies bedeutet, dass die „in good health“-Variante, die das voraussichtliche Rentengeschehen sowie höhere AU-Zeiten einbindet, die Beitragsverläufe nicht besser vorhersagen kann bzw. nicht besser mit ihnen korrespondiert.

Auch bestehen für die einfachere Berechnung etwas höhere $\mathrm{Zu}$ sammenhänge mit Ausgangsvariablen zu Beginn bzw. vor der Rehabilitationsmaßnahme. Wesentliche Ausgangsmerkmale waren dabei, wie in anderen Studien, Alter, Status und Ausmaß der Krankschreibungen direkt vor der Reha sowie im 12-Monatszeitraum davor, Dauer der Arbeitslosigkeit im Jahr vor der Reha sowie darüber hinaus auch die Eigeninitiative zur Reha sowie die subjektiv eingeschätzte Leistungsfähigkeit vor der Reha. Ähnliche, wenngleich moderatere Werte ergeben sich für die Vorhersage der RTW's aufgrund anderer Outcome-Kriterien wie der patientenseitigen Einschätzung des gesundheitlichen Befindens, des Ausmaßes an Beschwerden sowie der direkten Einschätzung von Veränderungen zum Katamnesezeitpunkt. Dabei liegt die Varianzaufklärung zwischen 16 und 22\%, wobei hier keine Überlegenheit der einen oder anderen Variante ermittelt werden konnte. Das Klassifikationsergebnis hingegen war wiederum für die einfachere Berechnung besser.

Insgesamt erscheint die einfachere Operationalisierung von RTW der ,in good health“-Variante etwas überlegen zu sein. Insbesondere der in letzterer stark eingehende Aspekt der Intention zur Frühberentung sollte kritisch hinterfragt werden. In der vorliegenden Studie waren das mehr als ein Viertel der befragten Rehabilitanden. Hier sollte unter Einbeziehung längerer Zeiträume nach der Reha weiter untersucht werden, ob diese geäußerte Intention tatsächlich zu einer Antragstellung bzw. zu einer Frühberentung führt. Dies kann anhand der hier betrachteten Daten nicht ausreichend beantwortet werden, Analysen für den 2- und 3-Jahreszeitraum nach der Reha sind in Vorbereitung.

Für die Berechnung und vor allem den Vergleich von RTW-Quoten sollte in der rehabilitationswissenschaftlichen Forschung künftig die Validierung sowohl anhand konkreter Beitragsleistungen als auch anhand „Patient Reported Outcomes“ (PROs) eine stärkere Berücksichtigung finden. Die prognostische Bedeutung vor allem der Intention zu einer Frühberentung für die längerfristigen Beiträge aus Beschäftigung ist weiter zu untersuchen.

\section{Limitierungen}

Ein wesentliches Kennzeichen der Studie besteht in der als 1-Punkt-Erhebung durchgeführten Patientenbefragung, weshalb die Ausgangssituation weitgehend als retrospektive Prä-Messung erhoben wurde. Eine aufwändigere Verlaufserhebung mit echten prä-Messungen vor der Reha sowie Messungen direkt bei Behandlungsende hätten Erhebungsaufwand und Studienlaufzeit deutlich erhöht und wurden daher nicht realisiert. Die Güte bzw. der 
Tab. 5 Vorhersagbarkeit der RTW's (1,0) durch Ausgangsdaten, Binäre Logistische Regressionsanalyse.

\begin{tabular}{|c|c|c|c|c|c|c|c|c|}
\hline & \multicolumn{2}{|c|}{ RTW_ZP } & \multicolumn{2}{|c|}{ RTW_kumV } & \multicolumn{2}{|c|}{ RTW_ZPgh } & \multicolumn{2}{|c|}{ RTW_kumVgh } \\
\hline & OR & $p$ & $O R$ & $p$ & OR & $p$ & OR & $p$ \\
\hline Alter & 0,89 & $<0,001$ & 0,95 & $<0,001$ & 0,94 & $<0,001$ & 0,95 & $<0,001$ \\
\hline \multicolumn{9}{|l|}{ Geschlecht } \\
\hline Männlich ( $n=1230-1328)$ & 0,99 & 0,970 & 0,76 & 0,141 & 0,79 & 0,041 & 0,75 & 0,011 \\
\hline \multicolumn{9}{|l|}{ Schulabschluss } \\
\hline (noch) keinen $(n=43-46)$ & 0,66 & 0,606 & 1,03 & 0,969 & 0,99 & 0,986 & 0,90 & 0,847 \\
\hline Hauptschule $(n=1133-1232)$ & 0,95 & 0,936 & 2,48 & 0,195 & 1,21 & 0,653 & 1,39 & 0,426 \\
\hline Mittlere Reife/Polytech. Oberschule $(n=481-522)$ & 1,00 & 0,995 & 2,99 & 0,126 & 1,26 & 0,595 & 1,50 & 0,337 \\
\hline Fachabitur/Fachhochschulreife $(n=89-98)$ & 1,83 & 0,428 & 2,48 & 0,249 & 1,05 & 0,915 & 1,02 & 0,968 \\
\hline Allgemeine Hochschulreife/Abitur ( $n=71-77)$ & 4,89 & 0,067 & 13,74 & 0,008 & 1,81 & 0,244 & 1,97 & 0,173 \\
\hline Sonstiges $(n=24-27)$ & Ref. & & Ref. & & Ref. & & Ref. & \\
\hline Erstauftreten Krankheitsbeschwerden & 1,05 & 0,381 & 0,95 & 0,354 & 0,96 & 0,262 & 0,94 & 0,086 \\
\hline \multicolumn{9}{|l|}{ Diagnosegruppe } \\
\hline Onkologie $(n=213-226)$ & 1,89 & 0,026 & 1,43 & 0,271 & 1,20 & 0,394 & 1,02 & 0,927 \\
\hline Psychische Erkrankungen ( $n=221-243)$ & 1,28 & 0,374 & 1,01 & 0,967 & 1,44 & 0,067 & 1,38 & 0,104 \\
\hline Kardiologie $(n=234-253)$ & 1,40 & 0,207 & 1,03 & 0,917 & 1,15 & 0,482 & 1,06 & 0,778 \\
\hline Orthopädie $(n=839-899)$ & 1,57 & 0,024 & 1,44 & 0,122 & 1,37 & 0,031 & 1,26 & 0,109 \\
\hline Sonstige $(n=337-383)$ & Ref. & & Ref. & & Ref. & & Ref. & \\
\hline \multicolumn{9}{|l|}{ Krankschreibung direkt vor Reha } \\
\hline Nein $(n=944-1037)$ & 1,76 & 0,001 & 4,68 & $<0,001$ & 1,76 & 0,001 & 2,25 & $<0,001$ \\
\hline Krankschreibungstage 12 Mon. prä & 1,00 & 0,156 & 1,00 & $<0,001$ & 1,00 & 0,156 & 1,00 & $<0,001$ \\
\hline \multicolumn{9}{|l|}{ Arbeitslosigkeit 12 Monate vor der Reha } \\
\hline Gar nicht ( $n=1596-1761)$ & 22,97 & $<0,001$ & 37,30 & $<0,001$ & 11,00 & $<0,001$ & 10,193 & $<0,001$ \\
\hline 2 bis zu 5 Wochen $(n=60-72)$ & 9,71 & $<0,001$ & 12,53 & $<0,001$ & 6,84 & $<0,001$ & 5,97 & $<0,001$ \\
\hline 6 bis 25 Wochen $(n=66-82)$ & 5,74 & $<0,001$ & 6,77 & $<0,001$ & 7,41 & $<0,001$ & 5,93 & $<0,001$ \\
\hline 26 bis 52 Wochen $(n=101-113)$ & Ref. & & Ref. & & Ref. & & Ref. & \\
\hline Anzahl Arztbesuche prä & 0,99 & 0,257 & 1,00 & 0,488 & 0,99 & 0,070 & 0,99 & 0,041 \\
\hline Krankenhaustage 12 Mon. prä & 1,00 & 0,537 & 1,00 & 0,472 & 1,00 & 0,374 & 1,00 & 0,457 \\
\hline \multicolumn{9}{|l|}{ Belastung durch gesundheitliche Probleme } \\
\hline Extrem stark $(n=793-868)$ & 0,42 & 0,203 & 0,34 & 0,183 & 1,17 & 0,700 & 1,23 & 0,756 \\
\hline Stark $(n=910-983)$ & 0,56 & 0,391 & 0,56 & 0,471 & 1,32 & 0,492 & 1,26 & 0,558 \\
\hline Weniger stark $(n=107-115)$ & 0,35 & 0,139 & 0,43 & 0,331 & 1,00 & 0,991 & 0,99 & 0,981 \\
\hline Überhaupt nicht $(n=34-38)$ & Ref. & & Ref. & & Ref. & & Ref. & \\
\hline \multicolumn{9}{|l|}{ Reha auf eigenen Wunsch veranlasst } \\
\hline $\mathrm{Ja}(n=873-958)$ & 1,49 & 0,033 & 1,69 & 0,012 & 1,29 & 0,078 & 1,38 & 0,025 \\
\hline Eher ja $(n=175-195)$ & 1,15 & 0,613 & 1,79 & 0,071 & 1,11 & 0,628 & 1,27 & 0,236 \\
\hline Teilweise ( $n=339-361)$ & 0,94 & 0,766 & 1,33 & 0,258 & 1,18 & 0,324 & 1,38 & 0,056 \\
\hline Eher nein $(n=80-89)$ & 0,83 & 0,564 & 0,79 & 0,525 & 1,00 & 0,985 & 0,92 & 0,776 \\
\hline Nein $(n=369-401)$ & Ref. & & Ref. & & Ref. & & Ref. & \\
\hline \multicolumn{9}{|l|}{ Berufliche Leistungsfähigkeit vor Reha } \\
\hline Voll leistungsfähig ( $n=330-360)$ & 3,54 & $<0,001$ & 2,16 & 0,008 & 1,67 & 0,011 & 1,62 & 0,015 \\
\hline Eingeschränkt leistungsfähig ( $n=1119-1220)$ & 2,20 & $<0,001$ & 2,44 & $<0,001$ & 1,27 & 0,135 & 1,34 & 0,062 \\
\hline Gar nicht leistungsfähig ( $n=386-424)$ & Ref. & & Ref. & & Ref. & & Ref. & \\
\hline \multicolumn{9}{|l|}{ Belastung berufliche Situation vor der Reha } \\
\hline Stark belastend $(n=983-1073)$ & 3,17 & 0,005 & 3,71 & 0,001 & 1,29 & 0,445 & 1,17 & 0,626 \\
\hline Etwas belastend $(n=373-404)$ & 3,18 & 0,007 & 4,46 & 0,001 & 1,66 & 0,123 & 1,47 & 0,241 \\
\hline Teils-Teils $(n=315-338)$ & 1,97 & 0,113 & 2,19 & 0,073 & 1,27 & 0,465 & 1,18 & 0,619 \\
\hline Eher erfüllend ( $n=113-122)$ & 2,34 & 0,071 & 2,07 & 0,113 & 2,03 & 0,052 & 1,97 & 0,063 \\
\hline Sehr erfüllend ( $n=60-68)$ & Ref. & & Ref. & & Ref. & & Ref. & \\
\hline \multicolumn{9}{|l|}{ Zufriedenheit berufliche Situation vor der Reha } \\
\hline Sehr zufrieden $(n=202-215)$ & 1,53 & 0,178 & 0,84 & 0,624 & 1,37 & 0,208 & 1,17 & 0,535 \\
\hline Zufrieden $(n=610-661)$ & 1,55 & 0,073 & 1,15 & 0,630 & 1,49 & 0,051 & 1,43 & 0,077 \\
\hline Teils - Teils $(n=496-557)$ & 1,85 & 0,015 & 1,38 & 0,280 & 1,47 & 0,057 & 1,45 & 0,063 \\
\hline Eher unzufrieden ( $n=304-331)$ & 1,39 & 0,187 & 0,99 & 0,962 & 1,27 & 0,250 & 1,12 & 0,581 \\
\hline Sehr unzufrieden $(n=214-248)$ & Ref. & & Ref. & & Ref. & & Ref. & \\
\hline GBesc Beschwerden bei Aufnahme & 1,01 & 0,317 & 1,02 & 0,094 & 0,98 & 0,002 & 0,98 & 0,001 \\
\hline GB 10 Gesundheitliches Befinden bei Aufnahme & 1,01 & 0,272 & 1,03 & 0,008 & 1,00 & 0,819 & 1,00 & 0,946 \\
\hline Nagelkerkes $R 2$ & 0,392 & & 0,430 & & 0,281 & & 0,286 & \\
\hline Klassifikation (\% Richtige) & 85,1 & & 89,3 & & 69,1 & & 70,3 & \\
\hline
\end{tabular}

$n=$ Min - Max; Ref. $=$ Referenz 
Tab. 6 Vorhersagbarkeit der RTW's (1,0) durch Patient Reported Outcomes (PROs), Binäre Logistische Regressionsanalyse.

\begin{tabular}{|c|c|c|c|c|c|c|c|c|}
\hline & \multicolumn{2}{|c|}{ RTW_ZP } & \multicolumn{2}{|c|}{ RTW_kumV } & \multicolumn{2}{|c|}{ RTW_ZPgh } & \multicolumn{2}{|c|}{ RTW_kumVgh } \\
\hline & $O R$ & $p$ & OR & $p$ & OR & p & $O R$ & $p$ \\
\hline Patientenzufriedenheit ZUF8 & 0,96 & 0,069 & 0,97 & 0,178 & 0,98 & 0,185 & 0,98 & 0,310 \\
\hline Berufliche Orientierung der Klinik MBOR-P & 1,00 & 0,778 & 1,05 & 0,010 & 0,99 & 0,252 & 0,99 & 0,407 \\
\hline Gesundheitliches Befinden GB10 Post & 1,04 & 0,002 & 1,08 & $<0,001$ & 1,05 & $<0,001$ & 1,05 & $<0,001$ \\
\hline Gesundheitliche Beschwerden GBesc Post & 1,01 & 0,467 & 1,04 & 0,007 & 0,98 & 0,038 & 0,99 & 0,115 \\
\hline GB10 Effektstärke prä-post & 0,92 & 0,534 & 0,84 & 0,271 & 0,96 & 0,653 & 0,98 & 0,806 \\
\hline GBesc Effektstärke prä-post & 0,98 & 0,891 & 1,21 & 0,315 & 1,20 & 0,082 & 1,24 & 0,036 \\
\hline Skala zur direkten Veränderungsmessung VM8 & 1,09 & $<0,001$ & 1,12 & $<0,001$ & 1,05 & $<0,001$ & 1,06 & $<0,001$ \\
\hline Nutzenbeurteilung der Reha & 1,29 & 0,081 & 1,22 & 0,212 & 1,31 & 0,008 & 1,23 & 0,039 \\
\hline Nagelkerkes $\mathbf{R}^{2}$ & 0,158 & & 0,215 & & 0,188 & & 0,191 & \\
\hline Klassifikation (\% Richtige) & 87,9 & & 91,0 & & 67,0 & & 68,9 & \\
\hline
\end{tabular}

$n=$ Min-Max; Ref. $=$ Referenz

Nutzen von retrospektiven Prä-Messungen werden kontrovers diskutiert; sie werden meist als weniger valide als echte prä-post-Vergleiche angesehen; jedoch gibt es auch überzeugende Argumente gegen diese Auffassung [31].

\section{Danksagung}

\section{$\nabla$}

Wir danken allen Rehabilitanden, die sich bereit erklärt haben, an der Studie mitzuwirken. Des Weiteren danken wir allen Mitarbeitern der DRV Baden-Württemberg, die die Durchführung der Studie ermöglicht bzw. unterstützt haben sowie der SAMA-Geschäftsstelle in Stuttgart für die kompetente Koordinierung der Projektgruppe.

\section{Interessenkonflikt}

Die Vertreter der beteiligten Institute (RN, RK, GK, DK und JS) sind Auftragnehmer der von der DRV Baden-Württemberg unterstützten Studie.

Eine Co-Autorin (HM) ist eine leitende Mitarbeiterin der DRV Baden-Württemberg, je zwei der Autoren (MR-B und GM) sind Chefärzte sowie leitende Psychologen (UK, JR) von Verbundkliniken.

Institute

${ }^{1}$ GfQG - Gesellschaft für Qualität im Gesundheitswesen, Karlsruhe

${ }^{2}$ University of Ulm, Institut für Rehabilitationsmedizinische Forschung,

Bad Buchau

${ }^{3}$ Deutsche Rentenversicherung Baden-Württemberg, Sozialmedizinischer

Dienst/Reha-Management, Stuttgart

${ }^{4}$ Gesundheitszentrum Federsee, Schlossklinik Bad Buchau Abt.

Psychosomatik, Bad Buchau

${ }^{5}$ Salinen Klinik, Qualitätsmanagement, Bad Rappenau

${ }^{6}$ Facharztpraxis für Innere Medizin, Bad Bocklet

${ }^{7}$ Institut für sportmedizinische Prävention und Rehabilitation an der

Universität Mainz, Mainz

${ }^{8}$ Hochschule Bonn-Rhein-Sieg, Fachbereich Sozialversicherung, Hennef

\section{Literatur}

1 Streibelt $M$, Egner $U$. Eine systematische Übersichtsarbeit zu den Einflussfaktoren auf die berufliche Wiedereingliederung nach beruflichen Bildungsleistungen. Rehabilitation (Stuttg) 2013; 52: 111-118

2 Streibelt $M$, Egner $U$. Eine Meta-Analyse zum Einfluss von Stichprobe, Messmethode und Messzeitpunkt auf die berufliche Wiedereingliederung nach beruflichen Bildungsleistungen. Rehabilitation (Stuttg) 2012; 51: 398-404

3 Wasiak $R$, Young $A E$, Roessler RT et al. Measuring return to work. J Occup Rehabil 2007; 17: 766-781
4 Schultz IZ, Stowell AW, Feuerstein $M$ et al. Models of return to work for musculoskeletal disorders. J Occup Rehabil 2007; 17: 327-352

5 Young AE, Roessler RT, Wasiak $R$ et al. A developmental conceptualization of return to work. J Occup Rehabil 2005; 15: 557-568

6 Steenstra I, Irvin E, Heymans MW. Systematic review of prognostic factors for workers' time away from work due to acute low-back pain: an update of a systematic review: final report to Workers Compensation Board of Manitoba: Final report to Workers Compensatio Board of Manitoba. Toronto. 2011

7 de Boer AG, Taskila T, Tamminga SJ et al. Interventions to enhance return-to-work for cancer patients. Cochrane Database Syst Rev 2011; CD007569

8 Ervasti J, Vahtera J, Pentti J et al. Return to work after depression-related absence by employees with and without other health conditions: a cohort study. Psychosom Med 2015; 77: 126-135

9 Mehnert A, de Boer A, Feuerstein M. Employment challenges for cancer survivors. Cancer 2013; 119 (Suppl 11): 2151-2159

10 Steenstra IA, Lee H, de Vroome EMM et al. Comparing current definitions of return to work: a measurement approach. J Occup Rehabil 2012; 22: 394-400

11 Streibelt $M$, Bethge $M$. Effects of intensified work-related multidisciplinary rehabilitation on occupational participation: a randomized-controlled trial in patients with chronic musculoskeletal disorders. Int J Rehabil Res 2014; 37: 61-66

12 Vermeulen SJ, Anema JR, Schellart AJ et al. Cost-effectiveness of a participatory return-to-work intervention for temporary agency workers and unemployed workers sick-listed due to musculoskeletal disorders: design of a randomised controlled trial. BMC Musculoskelet Disord 2010; 11: 60

13 Morfeld M. Rückkehr an den Arbeitsplatz: Welche Bedeutung hat dieses Ziel und wie kann man seine Erreichung messen? In: DRV Bund., (Hrsg.). 20. Rehabilitationswissenschaftliches Kolloquium Nachhaltigkeit durch Vernetzung. Bd. 93. DRV Schriften. Berlin: DRV Bund; 2011: 223-225

14 Bürger W, Dietsche S, Morfeld $M$ et al. Multiperspektivische Einschätzungen zur Wahrscheinlichkeit der Wiedereingliederung von Patienten ins Erwerbsleben nach orthopädischer Rehabilitation - Ergebnisse und prognostische Relevanz. Rehabilitation (Stuttg) 2001; 40: 217-225

15 Morfeld M. Die Operationalisierung von „Rückkehr an den Arbeitsplatz“ als unterschätzte Herausforderung an die Abbildung der Ergebnisqualität. In: DRV Bund., (Hrsg.). Ergebnisqualität der medizinischen Rehabilitation der Rentenversicherung. Berlin: DRV Bund; 2009: 100-113

16 Bürger $W$. Stufenweise Wiedereingliederung nach orthopädischer Rehabilitation - Teilnehmer, Durchführung, Wirksamkeit und Optimierungsbedarf. Rehabilitation (Stuttg) 2004; 43: 152-161

17 Streibelt $M$, Blume $C$, Thren $K$ et al. Value of functional capacity evaluation information in a clinical setting for predicting return to work. Arch Phys Med Rehabil 2009; 90: 429-434

18 Bestmann A, Zollmann P. Operationalisierung des Ergebnisses von beruflichen Bildungsmaßnahmen - Potenzial der Routinedaten der Deutschen Rentenversicherung. In: DRV Bund., (Hrsg.). DVR-Schriften 2011. Bd. 932011: 225-227

19 Bestmann A. Datenquellen und Datenqualität der Reha-Statistik-Datenbasis. DRV Schriften 2008; 55: 35-46

20 Klosterhuis H, Zollmann P, Grünbeck P. Verlaufsorientierte Auswertungen zur Rehabilitation. Aktuelle Ergebnisse aus der Reha-Statistik-Datenbasis. Deutsche Rentenversicherung 2004; 59: 287-296 
21 Nübling $R$, Kaluscha $R$, Krischak $G$ et al. Ergebnisqualität medizinischer Rehabilitation: Zum Zusammenhang zwischen „Patient Reported Outcomes“ (PROs) und geleisteten Sozialversicherungsbeiträgen. Die Rehabilitation 2016, in press

22 Nübling R, Kaluscha R, Holstiege J et al. Analyse des Behandlungserfolgs in der Medizinischen Rehabilitation. Konsequenzen für das interne Qualitätsmanagement „Reha-QM-Outcome-Studie“ des Qualitätsverbundes Gesundheit und der DRV Baden-Württemberg. [Abschlussbericht]; 2015

23 Nübling R, Kaluscha R, Holstiege J et al. Die „Reha-OM-Outcome-Studie“ des Qualitätsverbundes Gesundheit und der DRV Baden-Württemberg - Methodik und ausgewählte Ergebnisse. Prävention und Rehabilitation 2015; 27: 77-94

24 Schmidt J, Steffanowski $R$, Nübling $R$ et al. Ergebnisqualität stationärer psychosomatischer Rehabilitation. Vergleich unterschiedlicher Evaluationsstrategien. Regensburg: Roderer; 2003

25 Deck R, Mittag O, Hüppe A et al. Index zur Messung von Einschränkungen der Teilhabe (IMET) - Erste Ergebnisse eines ICF-orientierten Assessmentinstruments. Praxis Klinische Verhaltensmedizin und Rehabilitation 2007; 76: 113-120

26 Nübling $R$, Schmidt J, Reichelt $W$ et al. Behandlungsergebnisse ein Jahr nach stationärer psychosomatischer Rehabilitation. Prävention und Rehabilitation 2013; 25: 150-165
27 Nübling $R$, Hafen $K$, Jastrebow J et al. Indikation zu psychotherapeutischen und psychosozialen Massnahmen im Rahmen stationärer medizinischer Rehabilitation. Regensburg: Roderer; 2004

28 Schmidt J, Lamprecht F, Wittmann WW. Zufriedenheit mit der stationären Versorgung. Entwicklung eines Fragebogens und erste Validitätsuntersuchungen. Psychother med Psychol 1989; 39: 248-255

29 Kriz D, Nübling R, Steffanowski A et al. Patientenzufriedenheit in der stationären medizinischen Rehabilitation: Psychometrische Prüfung des ZUF-8. Zeitschrift für Medizinische Psychologie 2008; 17: 67-79

30 Nübling $R$, Bürger $W$, Kaiser $U$ et al. Medizinisch-berufliche orientierte Rehabilitation aus Patientensicht (MBOR-P) - Entwicklung einer Kurzskala zur subjektiven Einschätzung der erwerbsbezogenen Schwerpunktsetzung einer Rehabilitationsklinik. In: DRV-Schriften., (Hrsg.). Berlin: Deutsche Rentenversicherung Bund; 2014: 138-141

31 Zweig MH, Campbell G. Receiver-operating characteristic (ROC) plots: a fundamental evaluation tool in clinical medicine. Clinical chemistry 1993; 39: 561-577

32 Blome C, Augustin M. Measuring change in quality of life: Bias in prospective and retrospective evaluation. Value in health: the journal of the International Society for Pharmacoeconomics and Outcomes Research 2015; 18: 110-115 